\title{
STABILITY IN DISTRIBUTION OF NONLINEAR SYSTEMS WITH TIME-VARYING DELAYS AND SEMI-MARKOVIAN SWITCHING
}

\author{
ZAIMING LIU ${ }^{1}$ and JUN PENG ${ }^{凶 1}$ \\ (Received 21 November, 2007; revised 24 June, 2008)
}

\begin{abstract}
There has recently been considerable interest in the stability of stochastic differential equations with Markovian switching, and a number of results have been achieved. However, due to the exponential sojourn time of Markovian chain at each state, there are many restrictions on existing results for practical application. In this paper, we explore the problem of stability in distribution of nonlinear systems with time-varying delays and semi-Markov switching. Unlike existing models, the new model takes into account noise, time-varying delays and semi-Markov switching. By means of stochastic analysis, functional analysis and inequality techniques, sufficient conditions are obtained to guarantee the stability of the systems concerned. The proposed results are new and extend existing ones.
\end{abstract}

2000 Mathematics subject classification: primary 34A45; secondary 34K10.

Keywords and phrases: stochastic differential equations, stability in distribution, delays, semi-Markovian switching.

\section{Introduction}

In recent years, there has been considerable interest in research into stochastic systems, since stochastic modeling has come to play an important role in many real systems [1, 11]. Among them, stability analysis of different stochastic systems has been a subject of intense activity in the literature [4, 6, 10, 15]. When performing computation, there are many stochastic perturbations that affect system stability. Hybrid systems with Markov switching have been used to model many practical systems, in which abrupt changes in structure and parameters may occur.

Stability is the most fundamental concept in modern control theory, and switching systems can be used to describe a wide range of physical and engineering systems in practice, hence the considerable attention devoted to the stability of stochastic

\footnotetext{
${ }^{1}$ Department of Mathematical Sciences, Central South University, Changsha, Hunan 410075, China; e-mail: pengjunlrx@yahoo.com.cn.

(C) Australian Mathematical Society 2009, Serial-fee code 1446-1811/09 \$16.00
} 
differential equations with Markovian switching. For example, Ji and Chizeck [8] and Martion [13] studied the stability of the following linear systems with Markovian jump parameters:

$$
\dot{x}(t)=A(r(t)) x(t),
$$

where $r(t)$ is a finite state Markov chain taking values in $S=\{1,2, \ldots, N\}$. Basak et al. [2] discussed stability in distribution for a semi-linear stochastic differential equation with Markovian switching of the form

$$
d X(t)=A(r(t)) X(t) d t+\sigma(X(t), r(t)) d B(t) .
$$

In practice, significant time delays, whether constant or time-varying, are ubiquitous in dynamic systems. For example, in the modeling of biological neural networks or artificial neural networks, it is necessary to take account of time delays inherent in the phenomena because of the finite processing speed of information. The stability of systems with delays has been extensively studied; see, for example, [3,9] and references therein.

In many applied problems in reliability, queuing theory, and so on, we use semi-Markov processes. The main advantage of these is to allow non-exponential distributions for transition between states and to generalize several kinds of stochastic processes. Hence it is necessary to develop methods for calculating the stability conditions based on a semi-Markov evolution of the system. However, to the best of authors' knowledge, most of the research deals with the Markov switching case, and work on the semi-Markov case is rare. Yet semi-Markovian jump systems operate under fewer restrictions and can be widely found and used in many real system applications. They are less conservative and more applicable. An early paper on this topic is by Hou et al. [5], but they study the simplest linear systems with phasetype semi-Markovian jump parameters. In this paper, we discuss the following more general nonlinear systems with phase-type semi-Markovian jump parameter and timevarying delays:

$$
d x(t)=\hat{f}(x(t), x(t-\tau(t)), t, \hat{r}(t)) d t+\hat{\sigma}(x(t), x(t-\tau(t)), t, \hat{r}(t)) d B(t) .
$$

Using a similar method to [5], we first transform the phase-type semi-Markovian systems to a Markovian one. The problem of stability in distribution is then solved by considering the system with Markovian switching. The Markovian switching structure allows the derivation of the stability gained by applying standard methods to the system of interest.

This paper is organized as follows. In Section 2, we recall the method used in [5] and transform the problem of semi-Markovian jump parameters into the discussion of stability of systems with Markovian switching. In Section 3, we establish Ito stochastic differential equations with time-varying delays and semi-Markovian switching. Section 4 gives some sufficient conditions in terms of Lyapunov functions for the stability of the semi-Markovian switching systems. Our conclusions are drawn in Section 5. 


\section{Phase-type semi-Markovian processes}

Consider a Markov process $r(t)$ on the state space $\{1,2, \ldots, N+1\}$, where the states $1,2, \ldots, N$ are transient and the state $N+1$ is absorbing. The infinitesimal generator is

$$
Q=\left(\begin{array}{cc}
T & T^{0} \\
\mathbf{0} & 0
\end{array}\right),
$$

where the $N \times N$ matrix $T=\left(T_{i j}\right)$ satisfies $T_{i i}<0, T_{i j} \geq 0, i \neq j$, while $T^{0}=$ $\left(T_{1}^{0}, T_{2}^{0}, \ldots, T_{N}^{0}\right)$ is a non-negative column vector, and $T I+T^{0}=0$ where $I$ denotes an $N$-dimensional column vector having $1 \mathrm{~s}$ as its components. The initial distribution vector is $\left(\mathbf{a}, a_{N+1}\right)$, where $\mathbf{a}=\left(a_{1}, a_{2}, \ldots, a_{N}\right), \mathbf{a} I+a_{N+1}=1$.

LEMMA 2.1 ([14]). The distribution of the time at which $r(t)$ is absorbed in $N+1$ is

$$
F(t)=1-\boldsymbol{a} \exp (T t) I, \quad t \geq 0 .
$$

Definition 1 ([14]). The state $r(t)$ reached at time $t$ is called the phase of the distribution $F(\cdot)$ at time $t$. The distribution $F(\cdot)$ defined in $(2.1)$ on $[0,+\infty)$ is called a continuous phase-type $(\mathrm{PH})$ distribution and $(\mathbf{a}, T)$ is called its representation of order $N$.

LEMma 2.2 ([14]). The family of PH distributions is dense in all the families of distribution on $[0,+\infty)$.

Definition 2. Let $E$ be a finite or countable set. A stochastic process $\hat{r}(t)$ on the state process $E$ is called a phase semi-Markov process or a denumerable phase semiMarkov process (when $E$ is finite, $\hat{r}(t)$ is also called a finite phase semi-Markov process), if the following conditions hold.

(1) The sample paths of $(\hat{r}(t), t<+\infty)$ are right-continuous step functions and have left-hand limits with probability 1 .

(2) Denote the $n$th jump point of the process $\hat{r}(t)$ by $\tau_{n}(n=0,1,2, \ldots)$, where $\tau_{0} \equiv 0<\tau_{1}<\tau_{2}<\cdots<\tau_{n}<\cdots, \tau_{n} \uparrow+\infty$. Then $\tau_{n}(n=0,1,2, \ldots)$ are Markovian with respect to the process $\hat{r}(t)$.

(3) $F_{i j}(t)=P\left(\tau_{n+1}-\tau_{n} \leq t \mid \hat{r}\left(\tau_{n}\right)=i, \hat{r}\left(\tau_{n+1}\right)=j\right)=F_{i}(t)(i, j \in E, t \geq 0)$ do not depend on $j$ and $n$.

(4) $F_{i}(t)(i \in E)$ is a phase-type distribution.

In [5], it was proved that a finite phase semi-Markov process can be transformed to a finite Markov chain.

Consider a class of stochastic linear systems with semi-Markovian jump parameters in a fixed probability space $(\Omega, \mathcal{F}, P)$ :

$$
\left\{\begin{array}{l}
\dot{x}=\hat{A}(\hat{r}(t)) x(t), \quad t \geq 0, \\
x(0)=x_{0},
\end{array}\right.
$$

where the initial state $x_{0}$ is a fixed non-random constant vector, $r(0)=r_{0}$ and $\hat{A}(i)$ $(i \in E)$ are known matrices. 
THEOREM 2.3 ([5]). System (2.2) is equivalent to the system

$$
\left\{\begin{array}{l}
\dot{x}=A(r(t)) x(t), \quad t \geq 0, \\
x(0)=x_{0}
\end{array}\right.
$$

where $r(t)$ is the associated Markov chain of $\hat{r}(t)$.

By methods similar to those of [5], we can prove that a nonlinear system with semi-Markovian switching is equivalent to the corresponding system with Markovian switching by defining $f$ and $\sigma$ as follows:

$$
f(\psi(i, k)):=\hat{f}(i), \quad \sigma(\psi(i, k)):=\hat{\sigma}(i) .
$$

We thus have the following lemma.

LEMMA 2.4. System (1.1) is equivalent to the system

$$
d x(t)=f(x(t), x(t-\tau(t)), t, r(t)) d t+\sigma(x(t), x(t-\tau(t)), t, r(t)) d B(t)
$$

with the initial conditions $x_{0}=\xi$, where $\xi \in C_{\mathcal{F}_{0}}^{b}\left([-\tau, 0] ; R^{n}\right)$ is independent of $r(t)$ and $B(t)$, and $r(t)$ is the associated Markov chain of $\hat{r}(t)$.

\section{Itô stochastic differential equations with time-varying delay and semi-Markovian switching}

Let $\left(\Omega, \mathcal{F},\{\mathcal{F}\}_{t \geq 0}, P\right)$ be a complete probability space with a filtration $\{\mathcal{F}\}_{t \geq 0}$ satisfying the usual conditions (that is, it is right continuous and $\mathcal{F}_{0}$ contains all $P$-null sets). $B(t)=\left(B_{1}(t), \ldots, B_{m}(t)\right)^{T}$ is an $m$-dimensional Brownian motion defined on $\left(\Omega, \mathcal{F},\{\mathcal{F}\}_{t \geq 0}, P\right)$. Let $t_{0} \in R_{+}=[0, \infty), \tau(t):\left[t_{0}, \infty\right) \rightarrow[0, \tau]$. $C\left[[-\tau, 0], R^{n}\right]$ denotes the family of all continuous $R^{n}$-valued functions $\varphi$ on $[-\tau, 0]$ with the norm $\|\varphi\|=\sup _{-\tau \leq \theta \leq 0}|\varphi(\theta)| .|\cdot|$ is the Euclidean norm in $R^{n}$ and $\left\|x_{t}\right\|=\sup _{-\tau \leq \theta \leq 0}|x(t+\theta)|$, where $x_{t}(\theta)=x(t+\theta)$. Denote by $C_{\mathcal{F}_{0}}^{b}\left[[-\tau, 0], R^{n}\right]$ the family of all bounded $\mathcal{F}_{0}$ measurable, $C\left[[-\tau, 0], R^{n}\right]$-valued random variables. Let $p>0, t \geq 0$, denote by $L_{\mathcal{F}_{t}}^{p}\left([-\tau, 0] ; R^{n}\right)$ the family of all $\mathcal{F}_{t}$ measurable, $C\left([-\tau, 0] ; R^{n}\right)$-valued random variables. $\varphi=\{\varphi(\theta) \mid-\tau \leq \theta \leq 0\}$, and $\sup _{-\tau \leq \theta \leq 0} E|\varphi(\theta)|^{p}<\infty$.

Consider the following Itô stochastic differential delay equations with semiMarkovian switching:

$$
d x(t)=\hat{f}(x(t), x(t-\tau(t), t, \hat{r}(t)) d t+\hat{\sigma}(x(t), x(t-\tau(t), t, \hat{r}(t)) d B(t)
$$

with initial conditions $x_{0}=\xi$, where $\xi \in C_{\mathcal{F}_{0}}^{b}\left([-\tau, 0] ; R^{n}\right)$ is independent of $\hat{r}(t)$ and $B(t)$.

By Lemma 2.4, we need only to consider System (2.3). Assume that

$$
\begin{gathered}
f: L_{\mathcal{F}_{t}}^{p}\left([-\tau, 0] ; R^{n}\right) \times L_{\mathcal{F}_{t}}^{p}\left([-\tau, 0] ; R^{n}\right) \times R_{+} \times S \rightarrow R^{n}, \\
\sigma: L_{\mathcal{F}_{t}}^{p}\left([-\tau, 0] ; R^{n}\right) \times L_{\mathcal{F}_{t}}^{p}\left([-\tau, 0] ; R^{n}\right) \times R_{+} \times S \rightarrow R^{n \times m} .
\end{gathered}
$$


In this paper we always assume that under some conditions (3.1) has a unique continuous solution $x(t, \xi)$ such that for all $t \geq 0$, for all $a \geq 0$, $E\left(\sup _{-\tau \leq s \leq t}|x(s, \xi)|^{a}\right)<\infty$. Further, assume that $f(0,0, t, i) \equiv 0$ and $\sigma(0,0, t, i)$ $\equiv 0$ for all $i \in S$. Then (3.1) has a trivial solution $x \equiv 0$.

Denote by $C^{2,1}\left(R^{n} \times[-\tau, \infty) \times S ; R_{+}\right)$the family of all non-negative functions $V(x, t, i)$ on $R^{n} \times[-\tau, \infty) \times S$ which are twice continuously differentiable with respect to $x$ and once differentiable with respect to $t$.

For any $(x, t, i) \in R^{n} \times[-\tau,+\infty) \times S$ and any $y, z \in R^{n}$, define an operator $L$ by

$$
\begin{aligned}
L V(x, t, i)= & V_{t}(x, t, i)+V_{x}(x, t, i) f(x, y, t, i) \\
& +\frac{1}{2} \operatorname{trace}\left[\sigma^{T}(x, y, t, i) V_{x x} \sigma(x, y, t, i)\right]+\sum_{j=1}^{N} \gamma_{i j} V(x, t, j),
\end{aligned}
$$

where

$$
\begin{gathered}
V_{t}(x, t, i)=\frac{\partial V(x, t, i)}{\partial t}, \\
V_{x}(x, t, i)=\left(\frac{\partial V(x, t, i)}{\partial x_{1}}, \frac{\partial V(x, t, i)}{\partial x_{2}}, \ldots, \frac{\partial V(x, t, i)}{\partial x_{n}}\right), \\
V_{x x}(x, t, i)=\left(\frac{\partial^{2} V(x, t, i)}{\partial x_{i} \partial x_{j}}\right)_{n \times n} .
\end{gathered}
$$

In what follows we consider the difference between two solutions of (2.3) starting from different initial values, namely

$$
\begin{aligned}
X^{\varphi, i}(t)-X^{\phi, i}(t)= & \varphi-\phi+\int_{0}^{t}\left[f\left(X^{\varphi, i}(s), X^{\varphi, i}(s-\tau(s)), r_{i}(s)\right)\right. \\
& \left.-f\left(X^{\phi, i}(s), X^{\phi, i}(s-\tau(s)), r_{i}(s)\right)\right] d s \\
& +\int_{0}^{t}\left[\sigma\left(X^{\varphi, i}(s), X^{\varphi, i}(s-\tau(s)), r_{i}(s)\right)\right. \\
& \left.-\sigma\left(X^{\phi, i}(s), X^{\phi, i}(s-\tau(s)), r_{i}(s)\right)\right] d B(s) .
\end{aligned}
$$

For a given function $U \in C^{2}\left(R^{n} \times S ; R_{+}\right)$, we define an operator $\mathcal{L} U: R^{n \times 4} \times S$ $\rightarrow R$ associated with (3.2) by

$$
\begin{aligned}
\mathcal{L} U(x, & \left.y, z_{1}, z_{2}, i\right) \\
= & \sum_{j=1}^{N} \gamma_{i j} U(x-y, j)+U_{x}(x-y, i)\left[f\left(x, z_{1}, i\right)-f\left(y, z_{2}, i\right)\right] \\
& +\frac{1}{2} \operatorname{trace}\left(\left[\sigma\left(x, z_{1}, i\right)-\sigma\left(y, z_{2}, i\right)\right]^{T} U_{x x}(x-y, i)\left[\sigma\left(x, z_{1}, i\right)-\sigma\left(y, z_{2}, i\right)\right]\right) .
\end{aligned}
$$

We conclude this section by defining the stability in distribution of System (3.1). Let $Y(t)$ denote the $C\left([-\tau, 0] ; R^{n}\right) \times S$-valued process $\left(X_{t}, r(t)\right)$. Then $Y(t)$ is a 
Markovian process. Let $p(t, \xi, i, d \zeta \times\{j\})$ denote the transition probability of the process $Y(t)$.

DEFInItion 3. The process $Y(t)$ is said to be stable in distribution if there exists a probability measure $\pi(\cdot \times \cdot)$ on $C\left([-\tau, 0] ; R^{n}\right) \times S$ such that its transition probability $p(t, \xi, i, d \zeta \times\{j\})$ converges weakly to $\pi(d \zeta \times\{j\})$ as $t \rightarrow \infty$ for every $(\xi, i)$ $\in C\left([-\tau, 0] ; R^{n}\right) \times S$.

Lemma 3.1 ([11]). Assume (H1) of Theorem 4.1 holds. Then

$$
\sup _{0 \leq t<\infty} E\left\|X_{t}^{\xi, i}\right\|^{2}<\infty, \quad \forall(\xi, i) \in C\left([-\tau, 0] ; R^{n}\right) \times S .
$$

LEMMA 3.2 ([11] Burkholder-Davis-Gundy inequality). There exists a universal constant $K_{p}$ for any $0<p<\infty$ such that for every continuous local martingale $M$ vanishing at 0 and any stopping time $\tau$,

$$
E\left(\sup _{0 \leq s \leq \tau}\left|M_{s}\right|^{p}\right) \leq K_{p}\left(E(M, M)_{\tau}\right)^{p / 2},
$$

where $(M, M)_{\tau}$ is the cross-variation of $M$ and, in particular,

$$
K_{p}= \begin{cases}\left(\frac{32}{p}\right)^{p / 2} & \text { if } 0<p<2 \\ 4 & \text { if } p=2 \\ \frac{p^{p+1}}{2(p-1)^{p-1}} & \text { if } p>2 .\end{cases}
$$

LEMMA 3.3 ([12]). For every $p>0$ and any compact subset $K$ of $R^{n}$,

$$
\sup _{(x, i) \in K \times S} E\left[\sup _{0 \leq s \leq t}\left|X^{x, i}(s)\right|^{p}\right]<\infty, \quad \forall t \geq 0 .
$$

LEMMA 3.4 ([16]). If $V \in C^{2,1}\left(R^{n} \times\left[t_{0}, \infty\right) \times S ; R^{+}\right)$, then for any stopping times $0 \leq t_{1} \leq t_{2}<+\infty$

$$
E\left(V\left(x\left(t_{2}\right), t_{2}, r\left(t_{2}\right)\right)\right)=E V\left(x\left(t_{1}\right), t_{1}, r\left(t_{1}\right)\right)+E\left(\int_{t_{1}}^{t_{2}} L V(x(s), s, r(s)) d s\right)
$$

as long as the integrations involved exist and are finite.

\section{Main results}

From Lemma 2.4, in order to explore the stability of System (3.1), we need only consider systems with Markovian switching. In what follows we provide some sufficient conditions in terms of Lyapunov functions for the stability of the Markovian switching system. 
THEOREM 4.1. Let $c_{1}, c_{2}, \beta$ be positive numbers and $\lambda_{1}>\lambda_{2} \geq 0, \lambda_{3}>\lambda_{4} \geq 0$. If:

(H1) there exists function $V(x, i) \in C^{2}\left(R^{n} \times S ; R_{+}\right)$and $w_{1}(x) \in C\left(R^{n}, R_{+}\right)$such that for all $(x, i) \in R^{n} \times S$,

$$
c_{1}|x|^{2} \leq V(x, i) \leq w_{1}(x)
$$

and for all $(x, y, i) \in R^{n} \times R^{n} \times S$,

$$
L V(x, y, i) \leq-\lambda_{1} w_{1}(x)+\lambda_{2} w_{1}(y)+\beta
$$

(H2) there exist positive numbers $c_{2}$ and $U(x, i) \in C^{2}\left(R^{n} \times S ; R_{+}\right), w_{2}(x) \in C$ $\left(R^{n} ; R\right)$ such that

$$
c_{2}|x|^{2} \leq w_{2}(x) \wedge U(x, i)
$$

and $\mathcal{L} U\left(x, y, z_{1}, z_{2}, i\right) \leq-\lambda_{3} w_{2}(x-y)+\lambda_{4} w_{2}\left(z_{1}-z_{2}\right)$ for all $x, y, z_{1}, z_{2}$, $\in R^{n}$ and $i \in S$;

(H3) there is an $\alpha>0$ such that for $x, y, \bar{x}, \bar{y} \in R^{n}$ and $i \in S$,

$$
\begin{aligned}
& (x-\bar{x})^{T}(f(x, y, i)-f(\bar{x}, \bar{y}, i))+10|\sigma(x, y, i)-\sigma(\bar{x}, \bar{y}, i)|^{2} \\
& \quad \leq \frac{\alpha\left(|x-\bar{x}|^{2}+|y-\bar{y}|^{2}\right)}{2},
\end{aligned}
$$

then the process $Y(t)=\left(X_{t}, r(t)\right)$ is stable in distribution.

PROOF. By definition we need to show that there is a probability measure $\pi(\cdot \times \cdot) \in$ $\mathcal{P}\left(C\left([-\tau, 0] ; R^{n}\right) \times S\right)$ such that for any $(\varphi, i) \in C\left([-\tau, 0] ; R^{n}\right) \times S$, the transition probabilities $\{p(t, \varphi, i, \cdot \times \cdot) \mid t \geq 0\}$ converge weakly to $\pi(\cdot \times \cdot)$. Recalling the fact that the weak convergence of probability measure is a metric concept (see [7, Proposition 2.5]), we therefore need to show that for any $(\varphi, i) \in C\left([-\tau, 0] ; R^{n}\right) \times S$,

$$
\lim _{t \rightarrow \infty} d_{L}(p(t, \varphi, i, \cdot \times \cdot), \pi(\cdot \times \cdot))=0 .
$$

To prove the above equations, we consider the following two steps.

STEP 1. We have to prove that for any $(\varphi, i) \in C\left([-\tau, 0] ; R^{n}\right) \times S$,

$$
\lim _{t \rightarrow \infty} d_{L}(p(t, \varphi, i, \cdot \times \cdot), p(t, 0,1, \cdot \times \cdot))=0 .
$$

For $t \geq \tau$ and $\theta \in[0, \tau]$, by Itô's formula (see [2, 12]) and (3.2),

$$
\begin{aligned}
& \left|X^{\varphi, i}(t-\theta)-X^{\phi, i}(t-\theta)\right|^{2} \\
& =\left|X^{\varphi, i}(t-\tau)-X^{\phi, i}(t-\tau)\right|^{2}+\sum_{j=1}^{N} \gamma_{i j} \int_{t-\tau}^{t-\theta}\left|X^{\varphi, i}(s)-X^{\phi, i}(s)\right|^{2} d s \\
& \quad+\int_{t-\tau}^{t-\theta}|\sigma(A(\varphi, s))-\sigma(A(\phi, s))|^{2} d s
\end{aligned}
$$




$$
\begin{aligned}
& +2 \int_{t-\tau}^{t-\theta}\left(X^{\varphi, i}(s)-X^{\phi, i}(s)\right)^{T}(f(A(\varphi, s))-f(A(\phi, s))) d s \\
& +2 \int_{t-\tau}^{t-\theta}\left(X^{\varphi, i}(s)-X^{\phi, i}(s)\right)^{T}(\sigma(A(\varphi, s))-\sigma(A(\phi, s))) d B(s),
\end{aligned}
$$

where $F(A(\Phi, s))=F\left(X^{\Phi, i}(s), X^{\Phi, i}(s-\tau), r_{i}(s)\right)$ for $F=f, \sigma$ and $\Phi=\phi, \varphi$.

Using the Burkholder-Davis-Gundy inequality, we get

$$
\begin{aligned}
E \sup _{0 \leq \theta \leq \tau}\left|\int_{t-\tau}^{t-\theta}\left(X^{\varphi, i}(s)-X^{\phi, i}(s)\right)^{T}(\sigma(A(\varphi, s))-\sigma(A(\phi, s))) d B(s)\right| \\
\leq \frac{1}{4} E \sup _{0 \leq \theta \leq \tau}\left|X^{\varphi, i}(t-\theta)-X^{\phi, i}(t-\theta)\right|^{2} \\
\quad+8 E \int_{t-\tau}^{t}|g(A(\varphi, s))-g(A(\phi, s))|^{2} d s .
\end{aligned}
$$

Substituting (4.2) into (4.1) and using (H3), we obtain

$$
\begin{aligned}
E \sup _{0 \leq \theta \leq \tau} & \left|X^{\varphi, i}(t-\theta)-X^{\phi, i}(t-\theta)\right|^{2} \\
\leq & 2 E\left|X^{\varphi, i}(t-\tau)-X^{\phi, i}(t-\tau)\right|^{2} \\
& \quad+2 \alpha E \int_{t-\tau}^{t}\left(\left|X^{\varphi, i}(s)-X^{\phi, i}(s)\right|^{2}+\left|x^{\varphi, i}(s-\tau)-X^{\phi, i}(s-\tau)\right|^{2}\right) d s .
\end{aligned}
$$

Let $N$ be positive number and define the stopping time

$$
\tau_{N}=\inf \left\{t>0:\left|X^{\varphi, i}(t)-X^{\phi, i}(t)\right| \geq N\right\} .
$$

Setting $T_{N}=\tau_{N} \wedge t$ and applying the Itô formula to (3.2),

$$
\begin{aligned}
E U\left(X^{\varphi, i}\left(T_{N}\right)-X^{\phi, i}\left(T_{N}\right), r_{i}\left(T_{N}\right)\right) & \\
\leq & U(\varphi(0)-\phi(0), i)+\lambda_{4} \tau \int_{-\tau}^{0} w_{2}(\varphi(s)-\phi(s)) d s \\
& -\left(\lambda_{3}-\lambda_{4}\right) E \int_{0}^{T_{N}}\left|X^{\varphi, i}(s)-X^{\phi, i}(s)\right|^{2} d s .
\end{aligned}
$$

This implies that

$$
\begin{aligned}
\int_{0}^{\infty} & E\left|X^{\varphi, i}(s)-X^{\phi, i}(s)\right|^{2} d s \\
& \leq U(\varphi(0)-\phi(0), i)+\lambda_{4} \tau \int_{-\tau}^{0} w_{2}(\varphi(s)-\phi(s)) d s<\infty
\end{aligned}
$$

so that

$$
\lim _{t \rightarrow \infty} E\left|X^{\varphi, i}(t)-X^{\phi, i}(t)\right|^{2}=0
$$


[9] Stability in distribution of a class of delayed nonlinear systems with semi-Markovian switching 39

We now observe from (4.4) and (H2) that for any $\varepsilon>0$, there exists a $\delta_{1}>0$ such that

$$
E\left|X^{\varphi, i}(t)-X^{\phi, i}(t)\right|^{2}<\frac{\varepsilon}{3} \quad \text { if } \quad|\varphi-\phi|<\delta_{1} .
$$

Since $K$ is compact, there exist $\varphi_{1}, \varphi_{2}, \ldots, \varphi_{k}$ such that $\bigcup_{j=1}^{k} \rho\left(\varphi_{j}, \delta_{1}\right) \supseteq K$ where $\rho\left(\varphi_{i}, \delta_{1}\right)=\left\{\varphi \in C\left([-\tau, 0] ; R^{n}\right):\left\|\varphi-\varphi_{i}\right\|<\delta_{1}\right\}$. By (4.5), there exists a $T>0$ such that if $t \geq T$ and $1 \leq u, v \leq k$, then

$$
E\left|X^{\varphi_{u}, i}(t)-X^{\varphi_{v}, i}(t)\right|^{2}<\frac{\varepsilon}{3} .
$$

For any $\varphi, \phi \in K$, we can find $m, n$ such that $\varphi \in \rho\left(\varphi_{m}, \delta_{m}\right), \phi \in \rho\left(\varphi_{n}, \delta_{1}\right)$. By (4.6) and (4.7), we derive

$$
\begin{aligned}
E\left|X^{\varphi, i}(t)-X^{\phi, i}(t)\right|^{2} \leq & 3\left[E\left|X^{\varphi, i}(t)-X^{\varphi_{m}, i}(t)\right|^{2}+E\left|X^{\varphi_{m}, i}(t)-X^{\varphi_{n}, i}(t)\right|^{2}\right. \\
& \left.+E\left|X^{\varphi_{n}, i}(t)-X^{\phi, i}(t)\right|^{2}\right]<\varepsilon T
\end{aligned}
$$

for all $t \geq T$. It follows that

$$
\lim _{t \rightarrow \infty} E\left|X^{\varphi, i}(t)-X^{\phi, i}(t)\right|^{2}=0 \quad \text { uniformly in } \varphi, \phi \in K .
$$

By (4.3) and (4.8),

$$
\lim _{t \rightarrow \infty} E\left\|X_{t}^{\varphi, i}-X_{t}^{\phi, i}\right\|^{2}=0 \quad \text { uniformly in } \varphi, \phi \in K
$$

for any compact subset $K$ of $C\left([-\tau, 0] ; R^{n}\right)$.

Now

$$
\begin{aligned}
\mid E f & \left(Y^{\varphi, i}(t)\right)-E f\left(Y^{\phi, j}(t)\right) \mid \\
& \leq E\left|f\left(Y^{\varphi, i}(t)\right)-f\left(Y^{\phi, j}(t)\right)\right| \\
& \leq E\left[I_{T_{i j} \leq M_{1}} E\left|f\left(X_{t-T_{i j}}^{\bar{\varphi}, k}, r_{k}\left(t-T_{i j}\right)\right)-f\left(X_{t-T_{i j}}^{\bar{\phi}, k}, r_{k}\left(t-T_{i j}\right)\right)\right|\right] \\
& \leq E\left[I_{T_{i j} \leq M_{1},\|\bar{\varphi}\| \leq M_{2},\|\bar{\phi}\| \leq M_{2}} E\left(\left\|X_{t-\left(T_{i j} \wedge M_{1}\right)}^{\bar{\varphi}, k}-X_{t-\left(T_{i j} \wedge M_{1}\right)}^{\bar{\phi}, k}\right\|\right)\right] .
\end{aligned}
$$

On the other hand, by (4.9), there exists a $T>M_{1}$ such that

$$
E\left\|X_{t-\left(T_{i j} \wedge M_{1}\right)}^{\bar{\varphi}, k}-X_{t-\left(T_{i j} \wedge M_{1}\right)}^{\bar{\phi}, k}\right\|^{2}<\varepsilon^{2}, \quad \forall t \geq T_{1},
$$

provided that $\|\bar{\varphi}\| \leq M_{2}$ and $\|\bar{\phi}\| \leq M_{2}$, and hence

$$
E\left\|X_{t-\left(T_{i j} \wedge M_{1}\right)}^{\bar{\varphi}, k}-X_{t-\left(T_{i j} \wedge M_{1}\right)}^{\bar{\phi}, k}\right\| \leq\left(E\left\|X_{t-\left(T_{i j} \wedge M_{1}\right)}^{\bar{\varphi}, k}-X_{t-\left(T_{i j} \wedge M_{1}\right)}^{\bar{\phi}, k}\right\|^{2}\right)^{1 / 2} \leq \varepsilon .
$$


Substituting this into (4.10) gives

$$
\left|E f\left(Y^{\varphi, i}(t)\right)-E f\left(Y^{\phi, j}(t)\right)\right|<\varepsilon .
$$

Since $f \in L$ is arbitrary,

$$
\sup _{f \in L}\left|E f\left(Y^{\varphi, i}(t)\right)-E f\left(Y^{\phi, j}(t)\right)\right|<\varepsilon,
$$

that is,

$$
\lim _{t \rightarrow \infty} d_{L}(p(t, \varphi, i, \cdot \times \cdot), p(t, 0,1, \cdot \times \cdot))=0
$$

for any $(\varphi, i) \in C\left([-\tau, 0] ; R^{n}\right) \times S$.

STEP 2. We have to prove that $\{p(t, 0,1, \cdot \times \cdot) \mid t \geq 0\}$ is Cauchy in the space $\mathcal{P}\left(C\left([-\tau, 0] ; R^{n}\right) \times S\right)$ with metric $d_{L}$, that is, there is a unique $\pi(\cdot \times \cdot) \in$ $\mathcal{P}\left(C\left([-\tau, 0] ; R^{n}\right) \times S\right)$, such that

$$
\lim _{t \rightarrow \infty} d_{L}(p(t, 0,1, \cdot \times \cdot), \pi(\cdot \times \cdot))=0 .
$$

Let $\varphi \in C\left([-\tau, 0] ; R^{n}\right)$ and $i \in S$ be arbitrary. With $Y^{\varphi, i}(t)=\left(X_{t}^{\varphi, i}, r_{i}(t)\right)$, we need to show that

$$
\limsup _{t \rightarrow \infty f \in L} \mid E f\left(Y^{\varphi, i}(t+s)-E f\left(Y^{\varphi, i}(t)\right) \mid=0 .\right.
$$

Fix any $f \in L$. Using the Markov property,

$$
\begin{aligned}
\left|E f\left(Y^{\varphi, i}(t+s)\right)-E f\left(Y^{\varphi, i}(t)\right)\right| \\
\quad=\left|E\left[E\left(f\left(Y^{\varphi, i}(t+s)\right) \mid \mathcal{F}_{s}\right)\right]-E f\left(Y^{\varphi, i}(t)\right)\right| \\
\quad=\left|\sum_{m=1}^{N} \int E f\left(Y^{\phi, m}(t)\right) p(s, \varphi, i, d \phi \times\{m\})-E f\left(Y^{\varphi, i}(t)\right)\right| \\
\quad \leq \sum_{m=1}^{N} \int E\left|f\left(Y^{\phi, m}(t)\right)-f\left(Y^{\varphi, i}(t)\right)\right| p(s, \varphi, i, d \phi \times\{m\}),
\end{aligned}
$$

where the integration is over the whole space $C\left([-\tau, 0] ; R^{n}\right)$.

Let $\varepsilon>0$ be arbitrary. From Lemma 3.1, we know that there exists a compact subset $K$ of $C\left([-\tau, 0] ; R^{n}\right)$ such that

$$
p(s, \varphi, i, K \times S)>1-\varepsilon .
$$

Define the stopping time

$$
T_{m i}=\inf \left\{t>0 \mid r_{m}(t)=r_{i}(t)\right\}
$$


[11] Stability in distribution of a class of delayed nonlinear systems with semi-Markovian switching 41

By the ergodicity of the Markov chain, $T_{m i}<\infty$ almost surely, so there exists a positive number $M_{1}$ such that

$$
P\left(T_{m i}>M_{1}\right)<\varepsilon, \quad \forall m, i \in S .
$$

By the property of conditional expectation

$$
\begin{aligned}
& E\left|f\left(Y^{\phi, m}(t)\right)-f\left(Y^{\varphi, i}(t)\right)\right| \\
&= E\left(E\left[\left|f\left(X_{t}^{\phi, m}, r_{m}(t)\right)-f\left(X_{t}^{\varphi, i}, r(t)\right)\right| \mid \mathcal{F}_{T_{m i}}\right]\right) \\
& \leq E\left[I_{T_{m i} \leq M_{1}} E\left|f\left(X_{t-T_{m i}}^{\bar{\phi}, k}, r_{k}\left(t-T_{m i}\right)\right)-f\left(X_{t-T_{m i}}^{\bar{\varphi}, k}, r_{k}\left(t-T_{m i}\right)\right)\right|\right] \\
&+E\left[I_{T_{m i}>M_{1}} E\left|f\left(X_{t-T_{m i}}^{\bar{\phi}, k}, r_{k}\left(t-T_{m i}\right)\right)-f\left(X_{t-T_{m i}}^{\bar{\varphi}, k}, r_{k}\left(t-T_{m i}\right)\right)\right|\right] \\
& \leq E\left[I_{T_{m i} \leq M_{1}} E\left|f\left(X_{t-T_{m i}}^{\bar{\phi}, k}, r_{k}\left(t-T_{m i}\right)\right)-f\left(X_{t-T_{m i}}^{\bar{\varphi}, k}, r_{k}\left(t-T_{m i}\right)\right)\right|\right] \\
&+2 P\left(T_{m i}>M_{1}\right)
\end{aligned}
$$

where $k=r_{m}\left(T_{m i}\right)=r_{i}\left(T_{m i}\right), \bar{\phi}=X^{\phi, m}\left(T_{m i}\right), \bar{\varphi}=X^{\varphi, i}\left(T_{m i}\right)$.

By Lemma 3.3, there exists an $M_{2}>0$ such that for $\forall \phi \in K$,

$P\left(\omega: T_{m i} \leq M_{1},\|\bar{\varphi}\|>M_{2}\right)<\varepsilon \quad$ and $\quad P\left(\omega: T_{m i} \leq M_{1},\|\bar{\phi}\|>M_{2}\right)<\varepsilon$.

From (4.11)-(4.14) and the Markov property, for $t \geq M_{1}$,

$$
\begin{array}{rl}
\mid E & f\left(Y^{\varphi, i}(t+s)\right)-E f\left(Y^{\varphi, i}(t)\right) \mid \\
\leq & \sum_{m=1}^{N} \int_{K} E\left|f\left(Y^{\phi, m}(t)\right)-f\left(Y^{\varphi, i}(t)\right)\right| p(s, \varphi, i, d \phi \times\{m\})+\varepsilon \\
\leq & \sum_{m=1}^{N} \int_{K} E\left[I_{T_{m i} \leq M_{1}} E\left|f\left(X_{t-T_{m i}}^{\bar{\phi}, k}, r_{k}\left(t-T_{m i}\right)\right)-f\left(X_{t-T_{m i}}^{\bar{\varphi}, k}, r_{k}\left(t-T_{m i}\right)\right)\right|\right] \\
& \times p(s, \varphi, i, d \phi \times\{m\})+2 \varepsilon \\
\leq & \sum_{m=1}^{N} \int_{K} E\left[I_{T_{m i} \leq M_{1},\left\|\bar{\phi} \leq M_{2},\right\| \bar{\varphi} \leq M_{2}} E\left(\| X_{t-\left(T_{m i} \wedge M_{1}\right)}-X_{t-\left(T_{m i} \wedge M_{1}\right)}^{\bar{\varphi}, k}\right)\right] \\
& \times p(s, \varphi, i, d \phi \times\{m\})+6 \varepsilon .
\end{array}
$$

On the other hand, by (4.9), there exists a $T>M_{1}$ such that

$$
E\left\|X_{t-\left(T_{m i} \wedge M_{1}\right)}^{\bar{\phi}, k}-X_{t-\left(T_{m i} \wedge M_{1}\right)}^{\bar{\varphi}, k}\right\|^{2}<\varepsilon^{2}, \quad \forall t \geq T_{1},
$$

provided that $\|\bar{\phi}\| \leq M_{2}$ and $\|\bar{\varphi}\| \leq M_{2}$, and hence

$$
E\left(\left\|X_{t-\left(T_{m i} \wedge M_{1}\right)}^{\bar{\phi}, k}-X_{t-\left(T_{m i} \wedge M_{1}\right)}^{\bar{\varphi}, k}\right\|\right) \leq\left(E\left\|X_{t-\left(T_{m i} \wedge M_{1}\right)}^{\bar{\phi}, k}-X_{t-\left(T_{m i} \wedge M_{1}\right)}^{\bar{\varphi}, k}\right\|^{2}\right)^{1 / 2} \leq \varepsilon .
$$

Substituting this into (4.15) yields

$$
\left|E f\left(Y^{\varphi, i}(t+s)\right)-E f\left(Y^{\varphi, i}(t)\right)\right| \leq \varepsilon, \quad \forall t \geq T, s>0 .
$$


Since $f$ is arbitrary, it follows that

$$
\limsup _{t \rightarrow \infty f \in L}\left|E f\left(Y^{\varphi, i}(t+s)\right)-E f\left(Y^{\varphi, i}(t)\right)\right|=0 .
$$

Hence $\{p(t, 0,1, \cdot \times \cdot): t \geq 0\}$ is Cauchy in the metric space $\mathcal{P}\left(C\left([-\tau, 0] ; R^{n}\right)\right.$ $\times S)$ with metric $d_{L}$ and there exists a unique $\pi(\cdot \times \cdot) \in \mathcal{P}\left(C\left([-\tau, 0] ; R^{n}\right) \times S\right)$ such that

$$
\lim _{t \rightarrow \infty} d_{L}(p(t, 0,1, \cdot \times \cdot), \pi(\cdot \times \cdot))=0
$$

Therefore

$$
\begin{aligned}
\lim _{t \rightarrow \infty} d_{L}(p(t, \varphi, i, \cdot \times \cdot), \pi(\cdot \times \cdot)) \leq & \lim _{t \rightarrow \infty}\left[d_{L}(p(t, \varphi, i, \cdot \times \cdot), p(t, 0,1, \cdot \times \cdot))\right. \\
& \left.+d_{L}(p(t, 0,1, \cdot \times \cdot), \pi(\cdot \times \cdot))\right]=0
\end{aligned}
$$

as required.

REMARK 1. Condition (H1) implies that $\sup _{0 \leq t<\infty} E\left\|X_{t}^{\xi, i}\right\|^{2}<\infty$, for all $(\xi, i)$ $\in C\left([-\tau, 0], R^{n}\right) \times S$. By the Chebyshev inequality, it is easy to see that this guarantees that for any $(\xi, i) \in C\left([-\tau, 0], R^{n}\right) \times S$, the family $\{p(t, \xi, i, d \zeta \times\{j\})$, $t \geq 0\}$ is tight. That is, for any $\epsilon>0$, there is a compact subset $K=K(\xi, i, \epsilon)$ of $C\left([-\tau, 0], R^{n}\right)$ such that

$$
P(t, \xi, i, K \times S) \geq 1-\epsilon .
$$

REMARK 2. The hypothesis in Theorem 4.1 about the growth of $f$ and $\sigma$ is not too restrictive because of, for instance, results due to Yuan [17], Hou [5] and Mao [12].

REMARK 3. When System (3.1) reduces to a one-dimensional stochastic differential delay equation with semi-Markov switching of the form

$$
d X(t)=\hat{f}(X(t), \hat{r}(t))+\hat{\sigma}(X(t-\tau(t)), \hat{r}(t)) d B(t)
$$

on $t \geq 0$, the discussion of the stability of this system is easier. Assume that the phase semi-Markovian process $\hat{r}(t)$ has two states, denoted by 1 and 2 . The sojourn time in the first state is exponentially distributed with parameter 1 . The sojourn time in the second state is distributed as Erlang(2), which can be interpreted as the sum of two independent exponential distributions with parameter 1 . The state space of $Z(t)=(\hat{r}(t), J(t))$ is clearly $G=((1,1),(2,1),(2,2))$ and $\varphi((1,1))=1$, $\varphi((2,1))=2, \varphi((2,2))=3$. Hence the infinitesimal generator of $\varphi(Z(t))$ is

$$
Q=\left(\begin{array}{ccc}
-1 & 1 & 0 \\
0 & -1 & 1 \\
1 & 0 & -1
\end{array}\right)
$$

Choosing appropriate $f$ and $\sigma$ as in [17], we can prove that the system is stable in distribution. So in this sense, we generalize [17] to the semi-Markov case. 
REMARK 4. When the system is linear, it reduces to the system in [5] and all the results in [5] apply also in this paper.

REMARK 5. Here, we only discuss the phase-type semi-Markov processes, that is, the sojourn time in state $i$ follows a PH distribution. But this is enough to describe the general semi-Markov processes, because according to [14], the family of PH distributions is dense in all the families of distribution on $[0, \infty)$. We may choose a $\mathrm{PH}$ distribution to simulate every probability distribution on $[0, \infty)$ to any accuracy.

\section{Conclusions}

In this paper, we consider the problem of stochastic stability for a class of nonlinear systems with time-varying delays and semi-Markov switchings. Unlike existing models, the new model takes into account noise, time-varying delays and semiMarkov switching. By means of stochastic analysis, functional analysis and inequality techniques, sufficient conditions are proposed to guarantee the stability in distribution of the system of interest. Our results are new, and extend or improve upon existing ones. Our methods are suitable also for general control and filtering problems.

\section{Acknowledgements}

This research was supported by a grant from the National Science Foundation of China (No. 10371133).

The authors are grateful to reviewers for their valuable comments and suggestions, which improved the presentation of the paper.

\section{References}

[1] L. Arnold, Stochastic differential equations: theory and applications (Wiley, New York, 1972).

[2] G. K. Basak, A. Bisi and M. K Ghosh, "Stability of a random diffusion with linear drift", J. Math. Anal. Appl. 202 (1996) 604-622.

[3] M. Basin, E. Sanchez and R. Martinez-Zuniga, "Optimal linear filtering for systems with multiple state and observation delays", Int. J. Innov. Comput., Inform. Control 3 (2007) 1309-1320.

[4] R. Z. Has'minskii, Stochastic stability of differential equations (Sijthoff and Noordhoff, Amsterdam, 1981).

[5] Z. Hou, J. Luo and P. Shi, "Stochastic stability of linear systems with semi-Markovian jump parameters", ANZIAM J. 46 (2005) 331-340.

[6] L. Hu, P. Shi and B. Huang, "Stochastic stability and robust control for sampled-data systems with Markovian jump parameters”, J. Math. Anal. Appl. 313 (2006) 504-517.

[7] N. Ikeda and S. Watanable, Stochastic differential equations and diffusion processes (NorthHolland, Amsterdam, 1981).

[8] Y. Ji and H. J. Chizeck, "Controllability, stability and continuous time Markovian jump linear quadratic control”, IEEE Trans. Automat. Control 35 (1990) 777-788.

[9] Magdi S. Mahmoud, Y. Shi and Hazem N. Nounou, "Resilient observer-based control of uncertain time-delay systems”, Int. J. Innov. Comput., Inform. Control 3 (2007) 407-418.

[10] X. Mao, Exponential stability of stochastic differential equations (Marcel Dekker, New York, 1994).

[11] X. Mao, Stochastic differential equations and their applications (Horwood, Chichester, 1997). 
[12] X. Mao, "Stability of stochastic differential equations with Markov switching", Stochastic Proc. Appl. 79 (1999) 45-67.

[13] M. Mariton, Jump linear systems in automatic control (Marcel Dekker, New York, 1990).

[14] T. Morozan, "Stability and control for linear systems with jump Markov perturbations", Stochastic Anal. Appl. 13 (1995) 91-110.

[15] P. Shi, Y. Xia, G. Liu and D. Rees, "On designing of sliding mode control for stochastic jump systems", IEEE Trans. Automat. Control 51 (2006) 97-103.

[16] A. V. Skorohod, Asymptotic methods in the theory of stochastic differential equations (American Mathematical Society, Providence, RI, 1989).

[17] C. Yuan, J. Zou and X. Mao, "Stability in distribution of stochastic differential delay equations with Markovian switching", Systems Control Lett. 50 (2003) 195-207. 